\title{
RATIO AND INVERSE MOMENTS OF MARSHALL-OLKIN EXTENDED BURR TYPE XII DISTRIBUTION BASED ON LOWER GENERALIZED ORDER STATISTICS
}

\author{
Devendra Kumar* \\ Department of Statistics Amity Institute of Applied Sciences \\ Amity University, Noida-201 303, India
}

\begin{abstract}
In this small note we have established some new explicit expressions for ratio and inverse moments of lower generalized order statistics for the Marshall-Olkin extended Burr type XII distribution. These explicit expressions can be used to develop the relationship for moments of ordinary order statistics, record statistics and other ordered random variable techniques. Further, a characterization result of this distribution has been considered on using the conditional moment of the lower generalized order statistics.
\end{abstract}

Keywords: Lower generalized order statistics, order statistics, record values, Marshall-Olkin extended Burr type XII distribution, ratio and inverse moments, characterization.

\section{Introduction}

The concept of generalized order statistics (gos) was introduced by Kamps (1995) as a general framework for models of ordered random variables. Moreover, many other models of ordered random variables, such as, order statistics, $k$-th upper record values, upper record values, progressively Type II censoring order statistics, Pfeifer records and sequential order statistics are seen to be particular cases of ${ }^{g O S}$. These models can be effectively applied, e.g., in reliability theory. However, random variables that are decreasingly ordered cannot be integrated into this framework. Consequently, this model is inappropriate to study, e.g. reversed ordered order statistic and lower record values models. Using the concept of ${ }^{\circ O S}$, Pawlas and Szynal (2001) introduced the concept of lower generalized order statistics (lgos) to enable a common approach to descending order statistics, which was further studied by Burkschat et al. (2003) with the name dual generalized order statistics. The lgos models enable us to study decreasingly ordered random variables like reversed order statistics, lower record values and lower Pfeirfer records, through a common approach below:

Suppose $X(1, n, m, k), \ldots, X(n, n, m, k),(k \geq 1, m$ is a real number), are $n$ lgos from an absolutely continuous cumulative distribution function $(c d f) \quad F(x)$ with probability density function $(p d f) f(x)$, if their joint $p d f$ is of the form

\footnotetext{
* author e-mail address: devendrastats@gmail.com
} 


$$
k\left(\prod_{j=1}^{n-1} \gamma_{j}\right)\left(\prod_{i=1}^{n-1}\left[F\left(x_{i}\right)\right]^{m} f\left(x_{i}\right)\right)\left[F\left(x_{n}\right)\right]^{k-1} f\left(x_{n}\right)
$$

on the cone $F^{-1}(1)>x_{1} \geq x_{2} \geq \ldots \geq x_{n}>F^{-1}(0)$,

where $\gamma_{j}=k+(n-j)(m+1)>0$ for all $j, 1 \leq j \leq n, k$ is a positive integer and $m \geq-1$.

If $m=0$ and $k=1$, then this model reduces to the $(n-r+1)-$ th order statistic, from the sample $X_{1}, X_{2}, \ldots, X_{n}$ and (1.1) will be the joint $p d f$ of $n$ order statistics. If $k=1$ and $m=-1$, then (1.1) will be the joint $p d f$ of the first $n$ record values of the identically and independently distributed (iid) random variables with $c d f \quad F(x)$ and corresponding $p d f$ $f(x)$.

In view of (1.1), the marginal $p d f$ of the $r$-th $\operatorname{lgos}, X^{*}(r, n, m, k), 1 \leq r \leq n$, is

$$
f_{X^{*}(r, n, m, k)}(x)=\frac{C_{r-1}}{(r-1) !}[F(x)]^{\gamma_{r}-1} f(x) g_{m}^{r-1}(F(x)),
$$

and the joint $p d f$ of $X^{*}(r, n, m, k)$ and $X^{*}(s, n, m, k), 1 \leq r<s \leq n$, is

$$
\begin{gathered}
f_{X^{*}(r, n, m, k), X^{*}(s, n, m, k)}(x, y)=\frac{C_{s-1}}{(r-1) !(s-r-1) !}[F(x)]^{m} f(x) g_{m}^{r-1}(F(x)) \\
\times\left[h_{m}(F(y))-h_{m}(F(x))\right]^{s-r-1}[F(y)]^{\gamma_{s}-1} f(y), \quad x>y,
\end{gathered}
$$

where

$$
C_{r-1}=\prod_{i=1}^{r} \gamma_{i}, \quad h_{m}(x)= \begin{cases}-\frac{1}{m+1} x^{m+1}, & m \neq-1 \\ -\ln x, & m=-1\end{cases}
$$

and

$$
g_{m}(x)=h_{m}(x)-h_{m}(1), \quad x \in[0,1) .
$$

Moments of order statistics play an important role in quality control testing and reliability to predict the failure of future items based on the times of few early failures. An application of the first moments of order statistics can be considered in calculating the L-moments which are in fact the linear combinations of the expected order statistics. See Hosking (1990) for details. The concept of recurrence relations has its own importance. They are useful in reducing the number of operations necessary to obtain a general form for the function under consideration. Furthermore, they are used in characterizing the distributions, which is an important area, permitting the identification of population distribution from the properties of the sample. Recurrence relations and identities have attained importance as it reduces the amount of direct computation, time and labour. 
Various developments on lgos and related topics have been studied by Pawlas and Szynal (2001), Ahsanullah (2004, 2005), Mbah and Ahsanullah (2007). Recurrence relations for single and product moment of lgos from the exponentiated Kumarswamy, exponentiated Pareto, exponentiated gamma and generalized exponential distributions are derived by Kumar (2011) and Khan and Kumar (2010, 2011a, 2011b) respectively. Kumar (2013a, b) established recurrence relations for marginal and joint moments generating function of lgos from extended type I generalized logistic distribution and generalized logistic distribution respectively. Keseling (1999) characterized some continuous distributions based on conditional distributions of gos. Bieniek and Szynal (2003) characterized some distributions via linearity of regression of gos. Cramer et al. (2004) gave a unifying approach on characterization via linear regression of ordered random variables.

\subsection{Marshall-Olkin extended Burr type XII distribution}

A set of family of distributions which might be useful for fitting data has been proposed by Burr (1942) as a modeling lifetime data or survival data, which has twelve different forms of cumulative distribution functions. Among those twelve distribution functions, Burr Type X and Burr Type XII received the maximum attention. These distributions has been extensively used in various fields such as, simulation, quantal response, approximation of distributions, and development of non-normal control charts, in reliability theory and survival analysis. A number of standard theoretical distributions are limiting forms of Burr distributions. Burr type XII distribution plays major role in the analyses of lifetime and survival data. Shao et al. (2004) studied the models for extended three parameter of Burr type XII distribution and used this distribution to model extreme event with application to flood frequency. The flexibility of Burr type XII distribution has been studied by Rodriguez (1977). The Burr type XII has been widely used in various fields of sciences, such as in actuarial science, forestry, ecoloxicology, reliability and survival analysis.

The construction of the Marshall-Olkin extended Burr type XII distribution is rather simple and was first proposed by Marshall and Olkin (1997). As a new family of distributions by adding a parameter to obtain new families of distributions in connection with more flexible and represent a wide range of behavior than the original distributions. This distribution relates to a number of distributions such as Burr type XII, Marshall - Olkin extended Lomax distribution and hence its applicability in real life situations is significant. Since then, the Marshall-Olkin distributions have been widely studied in statistics and many authors have developed various Marshall-Olkin type distributions based on some well known distributions. See, for example the Marshall-Olkin extended Pareto distribution with application by Ghitany (2005), the MarshallOlkin extended Weibull distribution by Ghitany et al. (2005), the Marshall-Olkin extended Lomax distribution by Ghitany et al. (2007), Marshall-Olkin Extended Uniform Distribution by Josea and Krishna (2011), Marshall-Olkin Extended Log-Logistic Distribution by Gui (2013), and so on.

The uniqueness of this study comes from the fact that we provide a comprehensive description ratio and inverse moments of lower generalized order statistics of this distribution with hope that they will attract wider applications in life time analysis.

The Marshall-Olkin extended Burr type XII distribution was introduced by Al-Saiari et al. (2014). He studied some properties and maximum likelihood estimation of the unknown parameters. 
A random variable $X$ is said to have Marshall-Olkin extended Burr type XII distribution if its $p d f$ is of the form

$$
f(x)=\frac{\alpha \beta \lambda x^{\beta-1}\left(1+x^{\beta}\right)^{-(\lambda+1)}}{\left[1-(1-\alpha)\left(1+x^{\beta}\right)^{-\lambda}\right]^{2}}, \quad x>0, \alpha, \beta, \lambda>0
$$

and the corresponding $c d f$ is

$$
F(x)=\frac{1-\left(1+x^{\beta}\right)^{-\lambda}}{1-(1-\alpha)\left(1+x^{\beta}\right)^{-\lambda}}, \quad x>0, \alpha, \beta, \lambda>0
$$

and the survival function is given by

$$
S(x)=\frac{\alpha\left(1+x^{\beta}\right)^{-\lambda}}{1-(1-\alpha)\left(1+x^{\beta}\right)^{-\lambda}}, \quad x>0, \alpha, \beta, \lambda>0 .
$$

Burr XII and Marshall-Olkin extended Lomax distributions are consider as a special case of Marshall-Olkin extended Burr type XII distribution when $\alpha=1$ and $\beta=1$ respectively.

The paper is organized as follows. Some new expressions of the inverse moments of lgos from Marshall-Olkin extended Burr type XII distribution are derived in Section 2. In Section 3, some explicit expressions of ratio moments of lgos are derived. In Section 4, we obtained a characterization result of this distribution by using conditional expectation of lgos. Section 5 end with the some applications.

\section{Relations for inverse moments}

In this Section, the explicit expressions for inverse moments of lgos are considered. For the Marshall-Olkin extended Burr type XII distribution as given in (1.5), the $j$-th moments of $X^{*}(r, n, m, k)$ is given as

$$
\begin{aligned}
E\left[X^{* j-\beta}(r, n, m, k)\right] & =\int_{0}^{\infty} x^{j-\beta} f_{X^{*}(r, n, m, k)}(x) d x \\
= & \frac{C_{r-1}}{(r-1) !} \int_{0}^{\infty} x^{j-\beta}[F(x)]^{\gamma_{r}-1} f(x) g_{m}^{r-1}(F(x)) d x
\end{aligned}
$$

Further, on using the binomial expansion, we can rewrite (2.1) as

$$
\begin{aligned}
E\left[X^{* j}(r, n, m, k)\right] & =\frac{C_{r-1}}{(r-1) !(m+1)^{r-1}} \sum_{u=0}^{r-1}(-1)^{u}\left(\begin{array}{c}
r-1 \\
u
\end{array}\right) \\
& \times \int_{0}^{\infty} x^{j-\beta}[F(x)]^{\gamma_{r-u}-1} f(x) d x .
\end{aligned}
$$

Now letting $t=F(x)$ in (2.2), we get 


$$
\begin{gathered}
E\left[X^{* j-\beta}(r, n, m, k)\right]=\frac{C_{r-1}}{(r-1) !(m+1)^{r}} \sum_{u=0}^{r-1} \sum_{p=0}^{\infty} \sum_{q=0}^{\infty} \sum_{l=0}^{\infty} \frac{(-1)^{u+l+(j / \beta)-1}(1-\alpha)^{l}}{p ! q ! l !}\left(\begin{array}{c}
r-1 \\
u
\end{array}\right) \\
\quad \times\left(\frac{p}{\lambda}\right) \frac{\Gamma\left(1-\frac{j}{\beta}+p\right) \Gamma\left(\frac{p}{\lambda}+q\right)}{\Gamma\left(1-\frac{j}{\beta}\right) \Gamma\left(\frac{p}{\lambda}+1-l\right)} B\left(\frac{k}{m+1}+n-r+u+\frac{l+q}{m+1}, 1\right) .
\end{gathered}
$$

Since

$$
\sum_{a=0}^{b}(-1)^{a}\left(\begin{array}{l}
b \\
a
\end{array}\right) B(a+k, c)=B(k, c+b)
$$

where $B(a, b)$ is the complete beta function. Therefore,

$$
\begin{aligned}
E\left[X^{* j-\beta}(r, n,\right. & m, k)]=\frac{C_{r-1}}{(r-1) !(m+1)^{r}} \sum_{p=0}^{\infty} \sum_{q=0}^{\infty} \sum_{l=0}^{\infty} \frac{(-1)^{l+(j / \beta)-1}(1-\alpha)^{l}}{p ! q ! l !}\left(\frac{p}{\lambda}\right) \\
& \times \frac{\Gamma\left(1-\frac{j}{\beta}+p\right) \Gamma\left(\frac{p}{\lambda}+q\right)}{\Gamma\left(1-\frac{j}{\beta}\right) \Gamma\left(\frac{p}{\lambda}+1-l\right)} B\left(\frac{k}{m+1}+n-r+\frac{l+q}{m+1}, r\right) \\
& =\sum_{p=0}^{\infty} \sum_{q=0}^{\infty} \sum_{l=0}^{\infty} \frac{(-1)^{l+(j / \beta)-1}(1-\alpha)^{l}}{p ! q ! l !}\left(\frac{p}{\lambda}\right) \frac{\Gamma\left(1-\frac{j}{\beta}+p\right) \Gamma\left(\frac{p}{\lambda}+q\right)}{\Gamma\left(1-\frac{j}{\beta}\right) \Gamma\left(\frac{p}{\lambda}+1-l\right)} \\
& \times \frac{1}{\prod_{i=1}^{r}\left(1+\frac{q+l}{\gamma_{i}}\right)} .
\end{aligned}
$$

\section{Special cases}

i) Putting $m=0, k=1$ in (2.5), we get moments of order statistics from Marshall-Olkin extended Burr type XII distribution as;

$$
E\left[X_{n-r+1: n}^{j-\beta}\right]=\frac{n !}{(n-r) !} \sum_{p=0}^{\infty} \sum_{q=0}^{\infty} \sum_{l=0}^{\infty} \frac{(-1)^{l+(j / \beta)-1}(1-\alpha)^{l}}{p ! q ! l !}\left(\frac{p}{\lambda}\right)
$$




$$
\times \frac{\Gamma\left(1-\frac{j}{\beta}+p\right) \Gamma\left(\frac{p}{\lambda}+q\right)}{\Gamma\left(1-\frac{j}{\beta}\right) \Gamma\left(\frac{p}{\lambda}+1-l\right)} \frac{\Gamma[n-r+1+q+l]}{\Gamma[n+1+q+l]} .
$$

If we replace $n-r+1$ by $r$ we get

$$
\begin{aligned}
E\left[X_{r: n}^{j-\beta}\right]= & \frac{n !}{(r-1) !} \sum_{p=0}^{\infty} \sum_{q=0}^{\infty} \sum_{l=0}^{\infty} \frac{(-1)^{l+(j / \beta)-1}(1-\alpha)^{l}}{p ! q ! l !}\left(\frac{p}{\lambda}\right) \\
& \times \frac{\Gamma\left(1-\frac{j}{\beta}+p\right) \Gamma\left(\frac{p}{\lambda}+q\right)}{\Gamma\left(1-\frac{j}{\beta}\right) \Gamma\left(\frac{p}{\lambda}+1-l\right)} \frac{\Gamma[r+q+l]}{\Gamma[n+1+q+l]}
\end{aligned}
$$

and at $r=n$

$$
\begin{aligned}
E\left[X_{n: n}^{j-\beta}\right] & =\sum_{p=0}^{\infty} \sum_{q=0}^{\infty} \sum_{l=0}^{\infty} \frac{(-1)^{l+(j / \beta)-1}(1-\alpha)^{l}}{p ! q ! l !}\left(\frac{p}{\lambda}\right) \\
& \times \frac{\Gamma\left(1-\frac{j}{\beta}+p\right) \Gamma\left(\frac{p}{\lambda}+q\right)}{\Gamma\left(1-\frac{j}{\beta}\right) \Gamma\left(\frac{p}{\lambda}+1-l\right)} \frac{n}{(n+q+l)} .
\end{aligned}
$$

ii) Putting $m=-1$ in (2.5), to get moments of $k$-th record value from Marshall-Olkin extended Burr type XII distribution as;

$$
\begin{gathered}
E\left[X^{* j-\beta}(r, n,-1, k)\right]=\sum_{p=0}^{\infty} \sum_{q=0}^{\infty} \sum_{l=0}^{\infty} \frac{(-1)^{l+(j / \beta)-1}(1-\alpha)^{l}}{p ! q ! l !}\left(\frac{p}{\lambda}\right) \\
\times \frac{\Gamma\left(1-\frac{j}{\beta}+p\right) \Gamma\left(\frac{p}{\lambda}+q\right)}{\Gamma\left(1-\frac{j}{\beta}\right) \Gamma\left(\frac{p}{\lambda}+1-l\right)} \frac{1}{\left(1+\frac{q+l}{k}\right)^{r}} .
\end{gathered}
$$

\section{Relations for ratio moments}

In this Section, the explicit expressions for ratio moments of lgos are considered. For Marshall-Olkin Burr type XII distribution, the ratio moments of $X^{*}(r, n, m, k)$ and $X^{*}(s, n, m, k)$ is given as

$$
E\left[X^{* i}(r, n, m, k) X^{* j-\beta}(s, n, m, k)\right]=\int_{0}^{\infty} \int_{0}^{x} x^{i} y^{j-\beta} f_{X^{*}(r, n, m, k) X^{*}(s, n, m, k)}(x, y) d x d y .
$$


On using (1.3) and binomial expansion, we have

$$
\begin{aligned}
E\left[X^{* i}(r, n, m, k) X^{* j-\beta}(s, n, m, k)\right] & \\
= & \frac{C_{s-1}}{(r-1) !(s-r-1) !(m+1)^{s-2}} \sum_{u=0}^{r-1} \sum_{v=0}^{s-r-1}(-1)^{u+v}\left(\begin{array}{c}
r-1 \\
u
\end{array}\right)\left(\begin{array}{c}
s-r-1 \\
v
\end{array}\right) \\
& \times \int_{0}^{\infty} x^{i}[F(x)]^{(s-r+u-v)(m+1)-1} f(x) I(x) d x, \quad x>y,
\end{aligned}
$$

where

$$
I(x)=\int_{0}^{x} y^{j-\beta}[F(y)]^{\gamma_{s-v}-1} f(y) d y .
$$

By setting $t=F(y)$ in (3.2), we obtain

$$
I(x)=\sum_{p=0}^{\infty} \sum_{q=0}^{\infty} \sum_{l=0}^{\infty} \frac{(-1)^{l+(j / \beta)-1}(1-\alpha)^{l}(p / \lambda)}{p ! q ! l !} \frac{\Gamma\left(1-\frac{j}{\beta}+p\right) \Gamma\left(\frac{p}{\lambda}+q\right)}{\Gamma\left(1-\frac{j}{\beta}\right) \Gamma\left(\frac{p}{\lambda}+1-l\right)} \frac{[F(x)]^{\gamma_{s-v}+q+l}}{\left[\gamma_{s-v}+q+l\right]} .
$$

On substituting the above expression of $I(x)$ in (3.1), we find that

$$
\begin{gathered}
E\left[X^{* i}(r, n, m, k) X^{* j-\beta}(s, n, m, k)\right]=\frac{C_{s-1}}{(r-1) !(s-r-1) !(m+1)^{s-2}} \\
\times \sum_{u=0}^{r-1 s-r-1} \sum_{v=0}^{\infty} \sum_{p=0}^{\infty} \sum_{q=0}^{\infty} \sum_{l=0}^{\infty} \frac{(-1)^{u+v+l+(j / \beta)-1}(1-\alpha)^{l}(p / \lambda)}{p ! q ! l !}\left(\begin{array}{c}
r-1 \\
u
\end{array}\right)\left(\begin{array}{c}
s-r-1 \\
v
\end{array}\right) \\
\quad \times \frac{\Gamma\left(1-\frac{j}{\beta}+p\right) \Gamma\left(\frac{p}{\lambda}+q\right)}{\Gamma\left(1-\frac{j}{\beta}\right) \Gamma\left(\frac{p}{\lambda}+1-l\right)\left(\gamma_{s-v}+q+l\right)} \int_{0}^{\infty} x^{i}[F(x)]^{\gamma_{r-u}+q+l-1} f(x) d x .
\end{gathered}
$$

Again by setting $z=F(x)$ in (3.3) and simplifying the resulting equation, we get

$$
\begin{array}{r}
E\left[X^{* i}(r, n, m, k) X^{* j-\beta}(s, n, m, k)\right]=\frac{C_{s-1}}{(r-1) !(s-r-1) !(m+1)^{s-2}} \\
\quad \times \sum_{p=0}^{\infty} \sum_{q=0}^{\infty} \sum_{l=0}^{\infty} \sum_{a=0}^{\infty} \sum_{t=0}^{\infty} \sum_{w=0}^{\infty} \frac{(-1)^{l+w+t+((1+j) / \beta)-1}(1-\alpha)^{l+t}}{p ! q ! l ! w ! t ! a !}\left(\frac{p+w}{\lambda}\right)
\end{array}
$$




$$
\begin{aligned}
& \times \frac{\Gamma\left(1-\frac{j}{\beta}+p\right) \Gamma\left(\frac{p}{\lambda}+q\right)}{\Gamma\left(1-\frac{j}{\beta}\right) \Gamma\left(\frac{p}{\lambda}+1-l\right)} \sum_{u=0}^{r-1}(-1)^{u}\left(\begin{array}{c}
r-1 \\
u
\end{array}\right) B\left(\frac{k}{m+1}+n-r+u+\frac{q+l+a+t}{m+1}, 1\right) \\
& \times \frac{\Gamma\left(\frac{i}{\beta}+1\right) \Gamma\left(\frac{w}{\lambda}+a\right)}{\Gamma\left(\frac{i}{\beta}+1-w\right) \Gamma\left(\frac{w}{\lambda}+1-t\right)} \sum_{v=0}^{s-r-1}(-1)^{v}\left(\begin{array}{c}
s-r-1 \\
v
\end{array}\right) B\left(\frac{k}{m+1}+n-s+v+\frac{q+l}{m+1}, 1\right) .
\end{aligned}
$$

On using relation (2.4) in (3.4), we get

$$
\begin{gathered}
E\left[X^{* i}(r, n, m, k) X^{* j-\beta}(s, n, m, k)\right]=\frac{C_{s-1}}{(r-1) !(s-r-1) !(m+1)^{s-2}} \\
\quad \times \sum_{p=0}^{\infty} \sum_{q=0}^{\infty} \sum_{l=0}^{\infty} \sum_{a=0}^{\infty} \sum_{t=0}^{\infty} \sum_{w=0}^{\infty} \frac{(-1)^{l+w+t-1+(i+j) / \beta}(1-\alpha)^{l+t}}{p ! q ! l ! w ! t ! a !}\left(\frac{p+w}{\lambda}\right) \\
\quad \times \frac{\Gamma\left(1-\frac{j}{\beta}+p\right) \Gamma\left(\frac{p}{\lambda}+q\right)}{\Gamma\left(1-\frac{j}{\beta}\right) \Gamma\left(\frac{p}{\lambda}+1-l\right)} \frac{\Gamma\left(\frac{i}{\beta}+1\right) \Gamma\left(\frac{w}{\lambda}+a\right)}{\Gamma\left(\frac{i}{\beta}+1-w\right) \Gamma\left(\frac{w}{\lambda}+1-t\right)} \\
\times B\left(\frac{k}{m+1}+n-r+\frac{q+l+a+t}{m+1}, r\right) B\left(\frac{k}{m+1}+n-s+\frac{q+l}{m+1}, s-r\right) .
\end{gathered}
$$

Which after simplification yields

$$
\begin{aligned}
E\left[X^{* i}(r, n,\right. & \left.m, k) X^{* j-\beta}(s, n, m, k)\right] \\
= & \sum_{p=0}^{\infty} \sum_{q=0}^{\infty} \sum_{l=0}^{\infty} \sum_{a=0}^{\infty} \sum_{t=0}^{\infty} \sum_{w=0}^{\infty} \frac{(-1)^{l+w+t-1+(1+j) / \beta-1}(1-\alpha)^{l+t}}{p ! q ! l ! w ! t ! s !}\left(\frac{p+w}{\lambda}\right) \\
& \times \frac{\Gamma\left(1-\frac{j}{\beta}+p\right) \Gamma\left(\frac{p}{\lambda}+q\right)}{\Gamma\left(1-\frac{j}{\beta}\right) \Gamma\left(\frac{p}{\lambda}+1-l\right)} \frac{\Gamma\left(\frac{i}{\beta}+1\right) \Gamma\left(\frac{w}{\lambda}+a\right)}{\Gamma\left(\frac{i}{\beta}+1-w\right) \Gamma\left(\frac{w}{\lambda}+1-t\right)}
\end{aligned}
$$




$$
\times \frac{1}{\prod_{\phi=1}^{r}\left(1+\frac{q+l+a+t}{\gamma_{\phi}}\right) \prod_{\psi=r+1}^{s}\left(1+\frac{q+l}{\gamma_{\psi}}\right)} .
$$

\section{Special cases}

i) Setting $m=0, k=1$ in (3.5), we get product moments of order statistics from MarshallOlkin Burr type XII distribution as

$$
\begin{aligned}
E\left[X_{n-r+1: n}^{i} X_{n-s+1: n}^{j}\right]=\frac{n !}{(n-s) !} \sum_{p=0}^{\infty} \sum_{q=0}^{\infty} \sum_{a=0}^{\infty} \sum_{t=0}^{\infty} \sum_{l=0}^{\infty} \sum_{w=0}^{\infty}(-1)^{l+t+w-1+(i+j) / \beta} \\
\times \frac{\left(\frac{p+w}{\lambda}\right)(1-\alpha)^{l+t}}{p ! q ! l ! t ! w ! a !} \frac{\Gamma\left(1-\frac{j}{\beta}+p\right) \Gamma\left(\frac{p}{\lambda}+q\right)}{\Gamma\left(1-\frac{j}{\beta}\right) \Gamma\left(\frac{p}{\lambda}+1-l\right)} \frac{\Gamma\left(\frac{i}{\beta}+1\right) \Gamma\left(\frac{w}{\lambda}+a\right)}{\Gamma\left(\frac{i}{\beta}+1-w\right) \Gamma\left(\frac{w}{\lambda}+1-t\right)} \\
\times \frac{\Gamma(n-r+1+l+q+a+t) \Gamma(n-s+1+q+l)}{\Gamma(n+1+q+l+a+t) \Gamma(n-r+1+q+l)} .
\end{aligned}
$$

Replace $n-s+1$ by $r$ and $n-r+1$ by $s$

$$
\begin{aligned}
E\left[X_{r: n}^{i} X_{s: n}^{j}\right] & =\frac{n !}{(r-1) !} \sum_{p=0}^{\infty} \sum_{q=0}^{\infty} \sum_{a=0}^{\infty} \sum_{t=0 l=0}^{\infty} \sum_{w=0}^{\infty} \sum_{w}^{\infty}(-1)^{l+t+w-1+(i+j) / \beta} \\
& \times \frac{\left(\frac{p+w}{\lambda}\right)(1-\alpha)^{l+t} \Gamma\left(1-\frac{j}{\beta}+p\right) \Gamma\left(\frac{p}{\lambda}+q\right)}{p ! q ! l ! t ! w ! a !} \frac{\Gamma\left(\frac{i}{\beta}+1\right) \Gamma\left(\frac{w}{\lambda}+a\right)}{\Gamma\left(1-\frac{j}{\beta}\right) \Gamma\left(\frac{p}{\lambda}+1-l\right)} \frac{\Gamma\left(\frac{i}{\beta}+1-w\right) \Gamma\left(\frac{w}{\lambda}+1-t\right)}{\Gamma(s+l+q+a+t) \Gamma(r+q+l)} \\
& \times \frac{\Gamma(n+1+q+l+a+t) \Gamma(s+q+l)}{\Gamma}
\end{aligned}
$$

ii) If $m=-1$ in (3.5), we get product moments of $k$-th record values from Marshall-Olkin Burr type XII distribution as

$$
\begin{aligned}
E\left[\left(Z_{r}^{(k)}\right)^{i}\left(Z_{s}^{(k)}\right)^{j}\right] & =\sum_{p=0}^{\infty} \sum_{q=0}^{\infty} \sum_{a=0}^{\infty} \sum_{t=0 l=0}^{\infty} \sum_{w=0}^{\infty} \sum_{w}^{\infty}(-1)^{l+t+w-1+(i+j) / \beta} \\
\times & \left.\frac{\left(\frac{p+w}{\lambda}\right)(1-\alpha)^{l+t}}{p ! q ! l ! t ! w ! a !} \frac{\Gamma\left(1-\frac{j}{\beta}+p\right) \Gamma\left(\frac{p}{\lambda}+q\right)}{\Gamma\left(1-\frac{j}{\beta}\right) \Gamma\left(\frac{p}{\lambda}+1-l\right)} \frac{\Gamma\left(\frac{i}{\beta}+1\right) \Gamma\left(\frac{w}{\lambda}+a\right)}{\beta}+1-w\right) \Gamma\left(\frac{w}{\lambda}+1-t\right) \\
& \times \frac{1}{\left(1+\frac{q+l+a+t}{k}\right)^{r}\left(1+\frac{q+l}{k}\right)^{s-r}} .
\end{aligned}
$$




\section{Characterization}

Let $X^{*}(r, n, m, k), r=1,2, \ldots, n$ be $g o s$ from a continuous population with $d f \quad F(x)$ and $p d f \quad f(x)$, then the conditional $p d f$ of $X^{*}(s, n, m, k)$ given $X^{*}(r, n, m, k)=x$, $1 \leq r<s \leq n$, in view of (1.3) and (1.2), is

$$
\begin{aligned}
f_{X^{*}(s, n, m, k) \mid X^{*}(r, n, m, k)}(y \mid x)=\frac{C_{s-1}}{(s-r-1) ! C_{r-1}} \\
\times \frac{\left[\left(h_{m}(F(y))-h_{m}(F(x))\right]^{s-r-1}[F(y)]^{\gamma_{s}-1}\right.}{[F(x)]^{\gamma_{r+1}}} f(y), x<y .
\end{aligned}
$$

Theorem 4.1: Let $X$ be a non negative random variable having an absolutely continuous distribution function $F(x)$ with $F(0)=0$ and $0<F(x)<1$ for all $x>0$, then

$$
\begin{aligned}
& E\left[X^{*}(s, n, m, k) \mid X^{*}(r, n, m, k)=x\right]=\sum_{p=0}^{\infty} \sum_{q=0}^{\infty} \sum_{l=0}^{\infty} \frac{(-1)^{p+q+(1 / \beta)}(1-\alpha)^{q}(p / \lambda)}{p ! q ! l !} \\
& \quad \times\left[\frac{\left(1+x^{\beta}\right)^{\lambda}-1}{\left(1+x^{\beta}\right)^{\lambda}-(1-\alpha)}\right]^{q+l} \frac{\Gamma\left(\frac{1}{\beta}+1\right) \Gamma\left(\frac{p}{\lambda}+l\right)}{\Gamma\left(\frac{1}{\beta}+1-p\right) \Gamma\left(\frac{p}{\lambda}+1-q\right)} \prod_{j=1}^{s-r}\left(\frac{\gamma_{r+j}}{\gamma_{r+j}+q+l}\right),
\end{aligned}
$$

if and only if

$$
F(x)=\frac{1-\left(1+x^{\beta}\right)^{-\lambda}}{1-(1-\alpha)\left(1+x^{\beta}\right)^{-\lambda}}, \quad x>0, \alpha, \beta, \lambda>0 .
$$

Proof: From (4.1), we have

$$
\begin{gathered}
E\left[X^{*}(s, n, m, k) \mid X^{*}(r, n, m, k)=x\right]=\frac{C_{s-1}}{(s-r-1) ! C_{r-1}(m+1)^{s-r-1}} \\
\times \int_{0}^{x} y\left[1-\left(\frac{F(y)}{F(x)}\right)^{m+1}\right]^{s-r-1}\left(\frac{F(y)}{F(x)}\right)^{\gamma_{s}-1} \frac{f(y)}{F(x)} d y .
\end{gathered}
$$

By setting $u=\frac{F(y)}{F(x)}$ from (1.5) in (4.3), we obtain

$$
\begin{aligned}
& E\left[X^{*}(s, n, m, k) \mid X^{*}(r, n, m, k)=x\right]=\frac{C_{s-1}}{(s-r-1) ! C_{r-1}(m+1)^{s-r-1}} \\
& \times \sum_{p=0}^{\infty} \sum_{q=0}^{\infty} \sum_{l=0}^{\infty} \frac{(-1)^{p+q+(1 / \beta)}(1-\alpha)^{q}(p / \lambda)}{p ! q ! l !}\left[\frac{\left(1+x^{\beta}\right)^{\lambda}-1}{\left(1+x^{\beta}\right)^{\lambda}-(1-\alpha)}\right]^{q+l}
\end{aligned}
$$




$$
\times \frac{\Gamma\left(\frac{1}{\beta}+1\right) \Gamma\left(\frac{p}{\lambda}+l\right)}{\Gamma\left(\frac{1}{\beta}+1-p\right) \Gamma\left(\frac{p}{\lambda}+1-q\right)} \int_{0}^{1} u^{\gamma_{s}+q+l-1}\left(1-u^{m+1}\right)^{s-r-1} d u
$$

Again by setting $t=u^{m+1}$ in (4.4) and simplifying the resulting expression, we derive the relation given in (4.2).

To prove sufficient part, we have from (4.1) and (4.2)

$$
\begin{gathered}
\frac{C_{s-1}}{(s-r-1) ! C_{r-1}(m+1)^{s-r-1}} \int_{0}^{x} y\left[(F(x))^{m+1}-(F(y))^{m+1}\right]^{s-r-1} \\
\times[F(y)]^{\gamma_{s}-1} f(y) d y=[F(x)]^{\gamma_{r+1}} H_{r}(x),
\end{gathered}
$$

where

$$
\begin{aligned}
H_{r}(x) & =\sum_{p=0}^{\infty} \sum_{q=0}^{\infty} \sum_{l=0}^{\infty} \frac{(-1)^{p+q+(1 / \beta)}(1-\alpha)^{q}(p / \lambda)}{p ! q ! l !}\left[\frac{\left(1+x^{\beta}\right)^{\lambda}-1}{\left(1+x^{\beta}\right)^{\lambda}-(1-\alpha)}\right]^{q+l} \\
& \times \frac{\Gamma\left(\frac{1}{\beta}+1\right) \Gamma\left(\frac{p}{\lambda}+l\right)}{\Gamma\left(\frac{1}{\beta}+1-p\right) \Gamma\left(\frac{p}{\lambda}+1-q\right)} \prod_{j=1}^{s-r}\left(\frac{\gamma_{r+j}}{\gamma_{r+j}+q+l}\right) .
\end{aligned}
$$

Differentiating (4.7) both sides with respect to $x$, we get

$$
\begin{gathered}
\frac{C_{s-1}[F(x)]^{m} f(x)}{(s-r-2) ! C_{r-1}(m+1)^{s-r-2}} \int_{0}^{x} y\left[(F(x))^{m+1}-(F(y))^{m+1}\right]^{s-r-2}[F(y)]^{\gamma_{s}-1} f(y) d y \\
=H_{r}^{\prime}(x)[F(x)]^{\gamma_{r+1}}+\gamma_{r+1} H_{r}(x)[F(x)]^{\gamma_{r+1}-1} f(x)
\end{gathered}
$$

Therefore,

$$
\frac{f(x)}{F(x)}=\frac{H_{r}^{\prime}(x)}{\gamma_{r+1}\left[H_{r+1}(x)-H_{r}(x)\right]}=\frac{\alpha \beta \lambda x^{\beta-1}\left(1+x^{\beta}\right)^{\lambda-1}}{\left[\left(1+x^{\beta}\right)^{\lambda}-(1-\alpha)\right]\left[\left(1+x^{\beta}\right)^{\lambda}-1\right]}
$$

which proves that

$$
F(x)=\frac{1-\left(1+x^{\beta}\right)^{-\lambda}}{1-(1-\alpha)\left(1+x^{\beta}\right)^{-\lambda}}, \quad x>0, \alpha, \beta, \lambda>0 .
$$

\section{Application}

Moments of order statistics play an important role in quality control testing and reliability to predict the failure of future items based on the times of few early failures. In this Section we suggest some application based on moments discussed in Section 2. 
i) Estimation: The moments of order statistics and record values given in Section 2 can be used to obtain the best linear unbiased estimate of the parameters of the Marshall-Olkin Burr type XII distribution.

ii) Characterization: The Marshall-Olkin Burr type XII distribution given in (1.5) can be characterized by using conditional expectation of generalized order statistics as Theorem 4.1.

\section{Acknowledgements}

The author would like to thank two unknown referees and the associate editor for many valuable suggestions which have helped to improve the paper significantly.

\section{References}

[1].Ahsanullah, M. (2004): A characterization of the uniform distribution by dual generalized order statistics. Comm. Statist. Theory Methods, 33, 2921-2928.

[2].Ahsanullah, M. (2005): On lower generalized order statistics and a characterization of power function distribution. Stat. Methods, 7, 16-28.

[3].Al-Saiari, A. Y., Baharith, L. A. and Mousa, S. A. (2014): Marshall-Olkin Extended Burr Type XII Distribution, International Journal of Statistics and Probability, 3, 78-84.

[4].Bieniek, M. and Szynal, D. (2003): Characterizations of distributions via linearity of regression of generalized order statistics. Metrika, 58, 259-271.

[5].Burkschat, M., Cramer, E. and Kamps, U. (2003): Dual generalized order statistics. Metron, LXI, 13-26.

[6].Burr, I.W. (1942): Cumulative frequency distribution, Annals of Mathematical Statistics, 13, 215-232.

[7].Cramer, E., Kamps, U. and Keseling, C. (2004): Characterization via linear regression of ordered random variables: A unifying approach. Comm. Statist. Theory Methods, 33, 2885-2911.

[8].Ghitany, M.E, Al-Awadhi, F.A. and Alkhalfan, L.A. (2007): Marshall-Olkin extended Lomax distribution and its application to censored data. Commun Stat Theory Methods, 36, 1855-1866.

[9].Ghitany, M.E. (2005): Marshall-Olkin extended Pareto distribution and its application. Int J Appl Math 18, 17-32.

[10].Ghitany, M.E., Al-Hussaini, E. K. and Al-Jarallah, R.A. ( 2005): Marshall-Olkin extended Weibull distribution and its application to censored data. J Appl Stat 32, 1025-1034.

[11].Gui, W. (2013): Marshall-Olkin Extended Log-Logistic Distribution and Its Application in Minification Processes, Applied Mathematical Sciences, 7, 39473961. 
[12].Hosking, J.R.M. (1990): L-Moments: Analysis and estimation of distributions using linear combinations of order statistics. J. R. Statist. Soc., B52, 105-124.

[13].Josea and Krishna (2011): Marshall-Olkin Extended Uniform Distribution, ProbStat Forum, 04, 78-88.

[14].Kamps, U. (1995): A concept of generalized order statistics. B.G. Teubner Stuttgart.

[15].Keseling, C. (1999): Conditional distributions of generalized order statistics and some characterizations. Metrika, 49, 27-40.

[16].Khan, R.U. and Kumar, D. (2010): On moments of generalized order statistics from exponentiated Pareto distribution and its characterization. Appl. Math. Sci. (Ruse), 4, 2711-2722.

[17].Khan, R.U. and Kumar, D. (2011a): Expectation identities of lower generalized order statistics from generalized exponential distribution and a characterization. Math. Methods Statist., 20, 150-157.

[18].Khan, R.U. and Kumar, D. (2011b): Lower generalized order statistics from exponentiated gamma distribution and its characterization. ProbStats Forum, 4, 1224.

[19].Kumar, D. (2011): Explicit expressions for moments of lower generalized order statistics from exponentiated Kumaraswamy distribution and its characterization. $J$. Appl. Probab. Statist. 6, 61-72.

[20].Kumar, D. (2013a): Moment generating functions of lower generalized order statistics from generalized logistic distribution and its characterization. Pacific Journal of Applied Mathematics, 5, 29-44.

[21].Kumar, D. (2013b): Relations for marginal and moments generating functions of extended type I generalized logistic distribution based on lower generalized order statistics and characterization Tamsui Oxford journal of Mathematical Science, 29, 219-238.

[22].Marshall, A.W., and Olkin, I. (1997). A new Method for Adding a parameter to a family of distributions with application to the exponential and Weibull families. Biometrika, 84, 641-652.

[23].Mbah, A.K. and Ahsanullah, M. (2007): Some characterization of the power function distribution based on lower generalized order statistics. Pakistan J. Statist., 23, 139-146.

[24].Pawlas, P. and Szynal, D. (2001): Recurrence relations for single and product moments of lower generalized order statistics from the inverse Weibull distribution. Demonstratio Math., XXXIV, 353-358.

[25].Ristic and Kundu (2015): Marshall-Olkin Generalized Exponential Distribution, In Press. 
[26].Rodriguez, N. (1977). A guide to the Burr type XII distributions. Biometrika, 64, 129-134

[27].Shao, Q., Wong, H., Xia, J., and Ip, W. C. (2004). Models for extremes using the extended three-parameter Burr XII system with application to flood frequency analysis. Hydrological Sciences Journal, 49, 685-702.

Received December 15, 2015; accepted January 10, 2016. 